CONFORMAL GEOMETRY AND DYNAMICS

An Electronic Journal of the American Mathematical Society

Volume 11, Pages 128-136 (August 14, 2007)

S $1088-4173(07) 00167-1$

\title{
CONSTRUCTING SUBDIVISION RULES FROM RATIONAL MAPS
}

\author{
J. W. CANNON, W. J. FLOYD, AND W. R. PARRY
}

\begin{abstract}
This paper deepens the connections between critically finite rational maps and finite subdivision rules. The main theorem is that if $f$ is a critically finite rational map with no periodic critical points, then for any sufficiently large integer $n$ the iterate $f^{\circ n}$ is the subdivision map of a finite subdivision rule. This enables one to give essentially combinatorial models for the dynamics of such iterates.
\end{abstract}

We are interested here in connections between finite subdivision rules and rational maps. Finite subdivision rules arose out of our attempt to resolve Cannon's Conjecture: If $G$ is a Gromov-hyperbolic group whose space at infinity is a 2 -sphere, then $G$ has a cocompact, properly discontinuous, isometric action on hyperbolic 3space. Cannon's Conjecture can be reduced (see, for example, the Cannon-Swenson paper [5]) to a conjecture about (combinatorial) conformality for the action of such a group $G$ on its space at infinity, and finite subdivision rules were developed to give models for the action of such a Gromov-hyperbolic group on the 2-sphere at infinity.

There is also a connection between finite subdivision rules and rational maps. If $\mathcal{R}$ is an orientation-preserving finite subdivision rule such that the subdivision complex $S_{\mathcal{R}}$ is a 2 -sphere, then the subdivision map $\sigma_{\mathcal{R}}$ is a critically finite branched map of this 2 -sphere. In joint work 3 with Kenyon we consider these subdivision maps under the additional hypotheses that $\mathcal{R}$ has bounded valence (this is equivalent to the subdivision map not having periodic critical points) and mesh approaching 0. In [3, Theorem 3.1] we show that if $\mathcal{R}$ is conformal (in the combinatorial sense), then the subdivision map $\sigma_{\mathcal{R}}$ is equivalent to a rational map. The converse follows from [4, Theorem 4.7]. While a finite subdivision rule is defined in terms of a map from the subdivision complex to itself, it is basically a combinatorial procedure for recursively subdividing polygonal 2-complexes. By establishing the equivalence between a rational map and a subdivision map, one is giving an essentially combinatorial description of the action of the rational map.

In this paper we consider the problem of when a rational map $f$ is equivalent to the subdivision map of a finite subdivision rule. Since a subdivision complex has only finitely many vertices, such a rational map must be critically finite. We specialize here to the case that $f$ has no periodic critical points. Our main theorem,

Received by the editors March 15, 2007.

2000 Mathematics Subject Classification. Primary 37F10, 52C20; Secondary 57M12.

Key words and phrases. Finite subdivision rule, rational map, conformality.

This work was supported in part by NSF research grants DMS-0104030 and DMS-0203902.

(C)2007 American Mathematical Society Reverts to public domain 28 years from publication 
which has also been proved by Bonk and Meyer [1] when $f$ has a hyperbolic orbifold (which includes all but some well-understood examples), is the following:

Theorem 1. Suppose that $f$ is a critically finite rational map with no periodic critical points. If $n$ is a sufficiently large positive integer, then $f^{\circ}$ is the subdivision map of a finite subdivision rule.

In Section 1 we give basic definitions about finite subdivision rules, and in Section 2 we give basic definitions about critically finite maps. Theorem 1 is proved in Section 3 . Finally, in Section 4 we give a few questions about realizing rational maps by subdivision maps of finite subdivision rules.

\section{Finite Subdivision RUles}

In this section we give some of the basic definitions about finite subdivision rules. For examples and more details, see 2 .

A finite subdivision rule $\mathcal{R}$ consists of (i) a subdivision complex $S_{\mathcal{R}}$, (ii) a subdivision $\mathcal{R}\left(S_{\mathcal{R}}\right)$ of $S_{\mathcal{R}}$, and iii) a subdivision map $\sigma_{\mathcal{R}}: \mathcal{R}\left(S_{\mathcal{R}}\right) \rightarrow S_{\mathcal{R}}$. The subdivision complex $S_{\mathcal{R}}$ is a finite CW complex which is the union of its closed 2-cells and which has the property that for each closed 2-cell $\tilde{t}$ in $S_{\mathcal{R}}$ there is a cell structure $t$ on a closed 2-disk such that $t$ has at least three vertices, all of the vertices and edges of $t$ are in the boundary of the 2-disk, and the characteristic map $\psi_{t}: t \rightarrow S_{\mathcal{R}}$ with image $\widetilde{t}$ restricts to a homeomorphism on each open cell. (The cell complex $t$ is called a tile type of $\mathcal{R}$. Similarly, one defines an edge type corresponding to each 1-cell of $S_{\mathcal{R}}$.) The subdivision map $\sigma_{\mathcal{R}}$ is a continuous cellular map such that restriction to each open cell is a homeomorphism.

One can use finite subdivision rules to recursively subdivide suitable 2-complexes. Suppose $\mathcal{R}$ is a finite subdivision rule. A 2-complex $X$ is an $\mathcal{R}$-complex if it is the union of its closed 2-cells and there is a continuous cellular map $f: X \rightarrow$ $S_{\mathcal{R}}$ (called the structure map) such that the restriction of $f$ to each open cell is a homeomorphism. If $X$ is an $\mathcal{R}$-complex with associated map $f$, then the cell structure on $\mathcal{R}\left(S_{\mathcal{R}}\right)$ pulls back under $f$ to give a subdivision $\mathcal{R}(X)$ of $X$. One recursively defines subdivisions $\mathcal{R}^{n}(X), n>0$, by $\mathcal{R}^{n+1}(X)=\mathcal{R}\left(\mathcal{R}^{n}(X)\right)$. In particular, the subdivision complex $S_{\mathcal{R}}$ is an $\mathcal{R}$-complex and the tile types are $\mathcal{R}$-complexes. The closed 2-cells of a 2-complex are called tiles.

Let $\mathcal{R}$ be a finite subdivision rule. Then $\mathcal{R}$ is orientation preserving if there is an orientation on the union of the open 2-cells of $S_{\mathcal{R}}$ such that the restriction of $\sigma_{\mathcal{R}}$ to each open 2-cell of $\mathcal{R}\left(S_{\mathcal{R}}\right)$ is orientation preserving. We say that $\mathcal{R}$ has bounded valence if there is an upper bound to the vertex valences of the complexes $\mathcal{R}^{n}\left(S_{\mathcal{R}}\right)$, $n>0$. If $\mathcal{R}$ has bounded valence, then for each $\mathcal{R}$-complex $X$ with bounded vertex valences there is an upper bound to the vertex valences of $\mathcal{R}^{n}(X), n>0$. The finite subdivision rule $\mathcal{R}$ has an edge pairing if $S_{\mathcal{R}}$ is a surface, and $\mathcal{R}$ has mesh approaching 0 if for each open cover $U$ of $S_{\mathcal{R}}$, there is a positive integer $n$ such that each tile of $\mathcal{R}^{n}\left(S_{\mathcal{R}}\right)$ is contained in an element of $U$. The finite subdivision rule $\mathcal{R}$ has mesh approaching 0 combinatorially if (i) every edge of $S_{\mathcal{R}}$ is properly subdivided in some subdivision $\mathcal{R}^{n}\left(S_{\mathcal{R}}\right)$ and (ii) if $e_{1}$ and $e_{2}$ are disjoint edges of a tile type $t$, then for some positive integer $n$ no tile of $\mathcal{R}^{n}(t)$ intersects $e_{1}$ and $e_{2}$. This condition is easier to check than that of mesh approaching 0 , and a finite subdivision rule with mesh approaching 0 combinatorially is weakly equivalent to one with mesh approaching 0 . 


\section{CRitically finite maps}

We give here some of the basic definitions about critically finite maps. These came up in Thurston's topological characterization of critically finite rational maps. For further information, see the Thurston notes [1], the Douady-Hubbard paper [6], the Pilgrim paper [8], or the Pilgrim monograph [9].

Let $f: S^{2} \rightarrow S^{2}$ be an orientation-preserving branched map. A point $x \in S^{2}$ is called a critical point of $f$ if $D_{f}(x)>1$, where $D_{f}(x)$ is the topological degree of $f$ at $x$. We denote the set of critical points of $f$ by $\Omega_{f}$ and call it the critical set of $f$. The forward orbit of the critical set is the postcritical set $P_{f}=\bigcup_{n>0} f^{\circ n}\left(\Omega_{f}\right)$. The map $f$ is critically finite if $f$ has degree at least two and $P_{f}$ is a finite set. Two critically finite branched maps $f, g: S^{2} \rightarrow S^{2}$ are equivalent if there is a homeomorphism $h: S^{2} \rightarrow S^{2}$ such that $h\left(P_{f}\right)=P_{g},\left.(h \circ f)\right|_{P_{f}}=\left.(g \circ h)\right|_{P_{f}}$, and $h \circ f$ is isotopic rel $P_{f}$ to $g \circ h$.

We put an orbifold structure on the domain $S^{2}$ of a critically finite branched map. Given $x \in S^{2}$, define $\nu_{f}: S^{2} \rightarrow \mathbb{Z}_{+} \cup\{\infty\}$ by

$$
\nu_{f}(x)=\operatorname{lcm}\left\{D_{g}(y): g(y)=x \text { and } g=f^{\circ n} \text { for some } n \in \mathbb{Z}_{+}\right\} .
$$

Let $\mathcal{O}_{f}$ be the orbifold $\left(S^{2}, \nu_{f}\right)$. A point $x \in \mathcal{O}_{f}$ with $\nu_{f}(x)>1$ is called a distinguished point or ramified point. These are the points in $P_{f}$. The orbifold $\mathcal{O}_{f}$ is hyperbolic if there is a hyperbolic structure on $\mathcal{O}_{f} \backslash P_{f}$ such that near a distinguished point $x$ the metric is that of a hyperbolic cone with cone angle $2 \pi / \nu_{f}(x)$ (or a cusp if $\left.\nu_{f}(x)=\infty\right)$. One can show that the orbifold $\mathcal{O}_{f}$ is hyperbolic if and only if the Euler characteristic $\chi\left(\mathcal{O}_{f}\right)=2-\sum_{x \in P_{f}}\left(1-\frac{1}{\nu_{f}(x)}\right)$ is negative.

Lemma 2. Let $f: S^{2} \rightarrow S^{2}$ be an orientation-preserving branched map, let $x \in S^{2}$, and let $x^{\prime}=f(x)$. Then $D_{f}(x) \nu_{f}(x)$ divides $\nu_{f}\left(x^{\prime}\right)$.

Proof. Let $n \in \mathbb{Z}_{+}$and $y \in S^{2}$, and suppose that $f^{\circ n}(y)=x$. Let $g=f^{\circ n}$ and let $g^{\prime}=f^{\circ(n+1)}$. Then $g^{\prime}(y)=x^{\prime}$ and

$$
D_{g^{\prime}}(y)=D_{f}(x) D_{g}(y) .
$$

Since $\nu_{f}(x)$ is defined as the least common multiple of all positive integers of the form $D_{g}(y)$ and $\nu_{f}\left(x^{\prime}\right)$ is defined as the least common multiple of a set of positive integers which contains all products of the form $D_{f}(x) D_{g}(y)$, it follows that $D_{f}(x) \nu_{f}(x)$ divides $\nu_{f}\left(x^{\prime}\right)$.

Now suppose that $f: \widehat{\mathbb{C}} \rightarrow \widehat{\mathbb{C}}$ is a critically finite rational map. If $f$ has a periodic critical point, then the Fatou set is not empty. If $f$ doesn't have a periodic critical point, then $J_{f}=\widehat{\mathbb{C}}$ by Sullivan's classification [10] of the stable components of the Fatou set. If $\mathcal{O}_{f}$ is not hyperbolic, then it is one of the orbifolds $(\infty, \infty),(2,2, \infty)$, $(2,3,6),(2,4,4),(3,3,3)$, or $(2,2,2,2)$. In each case (see, for example, [6]), the possible rational maps are well understood.

Lemma 3. Let $f: \widehat{\mathbb{C}} \rightarrow \widehat{\mathbb{C}}$ be a critically finite rational map with no periodic critical points, and let $D$ be an open topological disk in $\widehat{\mathbb{C}} \backslash P_{f}$. Then for every $\epsilon>0$ there exists a positive integer $N$ such that if $n$ is an integer with $n \geq N$, then the diameter of every component of $f^{-n}(D)$ is less than $\epsilon$ in the spherical metric. 
Proof. The rational function $f$ determines an orbifold $\mathcal{O}_{f}=\left(\widehat{\mathbb{C}}, \nu_{f}\right)$. Let $\mathcal{O}$ be the orbifold $\left(\widehat{\mathbb{C}}, \nu_{f}^{\prime}\right)$, where $\nu_{f}^{\prime}(x)=\nu_{f}(x)$ if $\nu_{f}(x)=1$ and $\nu_{f}^{\prime}(x)=2 \nu_{f}(x)$ if $\nu_{f}(x)>1$. Let $\widetilde{\mathcal{O}}$ be the universal covering orbifold of $\mathcal{O}$ with branched cover $p: \widetilde{\mathcal{O}} \rightarrow \mathcal{O}$.

We next show that there exists a map $g: \widetilde{\mathcal{O}} \rightarrow \widetilde{\mathcal{O}}$ which lifts $f^{-1}$; in other words, if $x \in \widetilde{\mathcal{O}}$, then $f(p(g(x)))=p(x)$. (The existence of such a lift is also given in [7. Section 19].) For this, let $\mathcal{O}^{\prime}=\mathcal{O} \backslash P_{f}$ and let $\mathcal{O}^{\prime \prime}=\mathcal{O} \backslash\left(P_{f} \cup f^{-1}\left(P_{f}\right)\right)$. Let $\widetilde{\mathcal{O}^{\prime}}=p^{-1}\left(\mathcal{O}^{\prime}\right)$, and let $\widetilde{\mathcal{O}^{\prime \prime}}=p^{-1}\left(\mathcal{O}^{\prime \prime}\right)$. Let $p^{\prime}$ denote the restriction of $p$ to $\widetilde{\mathcal{O}}^{\prime}$, and let $p^{\prime \prime}$ denote the restriction of $p$ to $\widetilde{\mathcal{O}}^{\prime \prime}$. Let $f^{\prime \prime}$ denote the restriction of $f$ to $\mathcal{O}^{\prime \prime}$. Then $p^{\prime}: \widetilde{\mathcal{O}}^{\prime} \rightarrow \mathcal{O}^{\prime}, p^{\prime \prime}: \widetilde{\mathcal{O}}^{\prime \prime} \rightarrow \mathcal{O}^{\prime \prime}, f^{\prime \prime}: \mathcal{O}^{\prime \prime} \rightarrow \mathcal{O}^{\prime}$, and $f^{\prime \prime} \circ p^{\prime \prime}: \widetilde{\mathcal{O}}^{\prime \prime} \rightarrow \mathcal{O}^{\prime}$ are covering projections. Consider the following diagram.

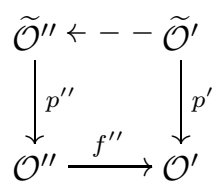

We wish to construct a map from $\widetilde{\mathcal{O}}^{\prime}$ to $\widetilde{\mathcal{O}}^{\prime \prime}$ which makes this diagram commute. After choosing base points compatibly, the lifting theorem for covering projections implies that such a map exists if and only if $p_{*}^{\prime}\left(\pi_{1}\left(\widetilde{\mathcal{O}}^{\prime}\right)\right) \subseteq\left(f^{\prime \prime} \circ p^{\prime \prime}\right)_{*}\left(\pi_{1}\left(\widetilde{\mathcal{O}}^{\prime \prime}\right)\right)$. The fundamental groups in question are free groups and this containment statement follows from the following divisibility statement: If $x, x^{\prime} \in \mathcal{O}$ and if $\widetilde{x}, \widetilde{x}^{\prime} \in \widetilde{\mathcal{O}}$ such that $f(x)=x^{\prime}, p(\widetilde{x})=x$, and $p\left(\widetilde{x}^{\prime}\right)=x^{\prime}$, then $D_{f \circ p}(\widetilde{x})$ divides $D_{p}\left(\widetilde{x}^{\prime}\right)$. But $D_{f \circ p}(\widetilde{x})=D_{f}(x) D_{p}(\widetilde{x})$,

$$
D_{p}(\widetilde{x})=\left\{\begin{array}{ll}
\nu_{f}(x) & \text { if } x \notin P_{f}, \\
2 \nu_{f}(x) & \text { if } x \in P_{f},
\end{array} \quad \text { and } \quad D_{p}\left(\widetilde{x}^{\prime}\right)= \begin{cases}\nu_{f}\left(x^{\prime}\right) & \text { if } x^{\prime} \notin P_{f}, \\
2 \nu_{f}\left(x^{\prime}\right) & \text { if } x^{\prime} \in P_{f} .\end{cases}\right.
$$

If $x^{\prime} \notin P_{f}$, then $x \notin P_{f}$; combining this with Lemma 2 shows that the statement is true and hence there does indeed exist a map from $\widetilde{\widetilde{\mathcal{O}}^{\prime}}$ to $\widetilde{\mathcal{O}}^{\prime \prime}$ which makes the diagram commute. This map easily extends to a map $g: \widetilde{\mathcal{O}} \rightarrow \widetilde{\mathcal{O}}$ which lifts $f^{-1}$.

Because $f$ has degree at least two and no periodic critical points, $\widetilde{\mathcal{O}}$ is hyperbolic. We next prove that $g$ is not a homeomorphism. Since $f$ has no periodic critical points, there must be a critical point $x$ of $f$ which is not a postcritical point. Let $\widetilde{x}$ be a lift of $x$ in $\widetilde{\mathcal{O}}$, let $y=f(x)$, and let $\widetilde{y}$ be a lift of $y$ in $\widetilde{\mathcal{O}}$. Then $D_{p}(\widetilde{x})=1$ and $D_{p}(\widetilde{y})=2 \nu_{f}(y)$. Since $D_{f \circ p}(\widetilde{x})=D_{f}(x) D_{p}(\widetilde{x})=D_{f}(x)$ and $D_{f}(x)$ divides $\nu_{f}(y)$ by Lemma 2, we see that $D_{f \circ p}(\widetilde{x})$ strictly divides $D_{p}(\widetilde{y})=D_{g}(\widetilde{y}) \cdot D_{f \circ p}(\widetilde{x})$. Hence $D_{g}(\widetilde{y})>1$ and $g$ is not injective in a neighborhood of $\widetilde{y}$. Hence $g$ strictly reduces distances in the hyperbolic metric. Because $\mathcal{O}$ is compact and $p: \widetilde{\mathcal{O}} \rightarrow \mathcal{O}$ is a regular branched cover, the group of covering transformations for $p$ acts cocompactly on $\widetilde{\mathcal{O}}$. This implies that $g$ reduces distances in the hyperbolic metric $d$ uniformly by a positive constant $c<1$; if $x, y \in \widetilde{\mathcal{O}}$, then $d(g(x), g(y)) \leq c d(x, y)$. So if $n$ is a positive integer and $\widetilde{D}$ is a lift of $D$ in $\widetilde{\mathcal{O}}$, then the diameter of $g^{n}(\widetilde{D})$ is at most $c^{n}$ times the diameter of $\widetilde{D}$. The lemma follows easily.

\section{THE MAIN THEOREM}

In this section we prove the main theorem, Theorem 1 We begin with a lemma that will be used in the proof. 
Lemma 4. Let $X$ be a closed topological disk with a regular tiling. If $u, v, w$ are distinct vertices of $X$, then there is an arc in the 1-skeleton of $X$ which has initial point $u$, has terminal point $w$, and contains $v$.

Proof. We prove this by induction on the number of tiles. If $X$ has a single tile, then it is a polygon and the result is clear. Suppose $n$ is a positive integer and the result holds if $X$ has a regular tiling with $n$ tiles. Let $Y$ be a closed topological disk with a regular tiling with $n+1$ tiles, and let $u, v, w$ be distinct vertices in $Y$. Since the tiling of $Y$ is regular, there are at least two tiles $t_{1}$ and $t_{2}$ such that $t_{i} \cap \partial Y$ is an arc. Let $t$ be one of these such that $v$ is in the closure $Y_{1}$ of $Y-t$. Then $Y_{1}$ is a closed topological disk with a regular tiling with $n$ tiles. By the choice of $t, v$ is in $Y_{1}$. If $u, v, w \in Y_{1}$, then it follows from induction that there is an arc in the 1-skeleton of $Y$ from $u$ to $w$ that passes through $v$. Now suppose that $u \notin Y_{1}$ but $w \in Y_{1}$. Let $u_{1} \neq w$ be one of the two points of $t \cap Y_{1} \cap \partial Y$. By induction there is an arc in $Y_{1}$ from $u_{1}$ to $w$ which passes through $v$, and it can easily be extended to an arc in $Y$ from $u$ to $w$ which passes through $v$. The case $u \in Y_{1}$ and $w \notin Y_{1}$ is symmetric, and the case that $u, w \notin Y_{1}$ is similar. This proves the lemma.

Proof of Theorem 1. Let $\delta>0$ such that the closures of the $\delta$ neighborhoods of the postcritical points are disjoint. We begin by choosing a simple closed curve $\alpha$ in $\widehat{\mathbb{C}}$ such that $\alpha$ contains $P_{f}$, for each $v \in P_{f} \alpha$ intersects $\overline{B_{\delta}(v)}$ in an arc, and for each $v \in P_{f} \alpha$ intersects $\overline{B_{\delta / 2}(v)}$ in an arc. We next construct a finite CW complex $S$ with underlying space $\widehat{\mathbb{C}}$ such that $P_{f}$ is the set of vertices of $S$ and $\alpha$ is the 1-skeleton of $S$. For each $n \in \mathbb{Z}_{+}$, let $f^{-n}(S)$ be the finite CW complex which is the pullback of $S$ under $f^{\circ n}$. Note that if $\alpha \subset f^{-1}(\alpha)$, then $S$ is the subdivision complex of a finite subdivision rule with subdivision map $f$, and hence we are done.

Now let $\epsilon>0$ so that $\epsilon<\delta / 2$, the distance between any two distinct components of $\alpha \backslash \bigcup_{v \in P_{f}} B_{\delta / 2}(v)$ is greater than $3 \epsilon$, and there is a point in $\widehat{\mathbb{C}}$ whose distance from $\alpha \cup\left(\bigcup_{v \in P_{f}} \overline{B_{\delta}(v)}\right)$ is greater than $3 \epsilon$. By Lemma 3 , there is a positive integer $N$ such that if $n \geq N$, then each tile of $f^{-n}(S)$ has diameter less than $\epsilon$. Let $n \in \mathbb{Z}_{+}$with $n \geq N$, and let $g=f^{\circ n}$. It is easy to see that $P_{g}=P_{f}$. In the next paragraph we construct a simple closed curve $\beta$ in the 1-skeleton of $f^{-n}(S)$ such that $\beta$ is isotopic rel $P_{f}$ to $\alpha$.

Choose an orientation for $\alpha$. Let $v_{1}, \ldots, v_{k}$ be an enumeration of the elements of $P_{g}$, labeled in cyclic order as they appear in $\alpha$, and for convenience let $v_{k+1}=v_{1}$. For each integer $i$ with $1 \leq i \leq k$, choose a subcomplex $D_{i}$ of $g^{-1}(S)$ such that $D_{i}$ is a closed topological disk, $B_{\delta / 2}\left(v_{i}\right) \subset D_{i}$, and $D_{i} \subset B_{\delta}\left(v_{i}\right)$. (For example, one could choose $D_{i}$ to be the union of the closed star of $B_{\delta / 2}\left(v_{i}\right)$ together with those complementary components that are contained in $B_{\delta}\left(v_{i}\right)$.) For each integer $i$ with $1 \leq i \leq k$, let $\alpha_{i}$ be the subarc of $\alpha$ from $v_{i}$ to $v_{i+1}$ (so $\operatorname{int}\left(\alpha_{i}\right) \cap \operatorname{int}\left(\alpha_{j}\right)=\emptyset$ if $i \neq j$ ), and let $\beta_{i}$ be an arc in the 1-skeleton of $g^{-1}(S)$ such that $\beta_{i} \cap D_{i}$ is a vertex $a_{i}, \beta_{i} \cap D_{i+1}$ is a vertex $b_{i}, a_{i}$ and $b_{i}$ are the endpoints of $\beta_{i}$, and $\beta_{i} \subset B_{\epsilon}\left(\alpha_{i}\right)$. For convenience we define $b_{0}=b_{k}$. The choice of $\epsilon$ guarantees that $b_{i} \neq a_{i+1}$ for $i \in\{0, \ldots, k-1\}$, and that $\beta_{i} \cap \beta_{j}=\emptyset$ if $i \neq j$. By Lemma 4, for each $i$ with $1 \leq i \leq k$ there is an arc $\gamma_{i}$ in the 1-skeleton of $D_{i}$ which joins $a_{i}$ and $b_{i-1}$ and passes through $v_{i}$. Then the union of the $\operatorname{arcs} \beta_{i}$ and $\gamma_{i}, 1 \leq i \leq k$, is the image of a simple closed curve $\beta$ in the 1-skeleton of $g^{-1}(S)$, and this curve is isotopic rel $P_{g}$ to $\alpha$. 
Since $\beta$ is isotopic to $\alpha$ rel $P_{g}$, there is an isotopy $H: \widehat{\mathbb{C}} \times I \rightarrow \widehat{\mathbb{C}}$ rel $P_{g}$ from the identity map on $\widehat{\mathbb{C}}$ to a homeomorphism $H_{1}: \widehat{\mathbb{C}} \rightarrow \widehat{\mathbb{C}}$ such that $H_{1}(\alpha)=\beta$. (Here we are following the convention that $H_{t}(x)=H(x, t)$.) Let $\mathcal{R}$ be the finite subdivision rule with subdivision complex $S_{\mathcal{R}}=\widehat{\mathbb{C}}$ such that the cells of $S_{\mathcal{R}}$ are the images under $H_{1}$ of the cells of $S$, and with subdivision map $\sigma_{\mathcal{R}}=H_{1} \circ g$. Note that if $x$ is a vertex of $\mathcal{R}\left(S_{\mathcal{R}}\right)=g^{-1}(S)$, then $g(x) \in P_{g}$ and so $\sigma_{\mathcal{R}}(x)=H_{1}(g(x))=g(x)$.

By the choices of $n$ and $\epsilon$, every edge of $S_{\mathcal{R}}$ is properly subdivided in $\mathcal{R}\left(S_{\mathcal{R}}\right)$ and, if $t$ is one of the two tile types, no tile of $\mathcal{R}(t)$ intersects two disjoint edges of $t$. Hence $\mathcal{R}$ has mesh approaching 0 combinatorially. If $\mathcal{R}$ does not have mesh approaching 0 , then by [2, Theorem 2.3] $\mathcal{R}$ is weakly isomorphic to a finite subdivision rule $\mathcal{Q}$ with mesh approaching 0 . Hence there are cellular homeomorphisms $\rho_{1}, \rho_{2}: S_{\mathcal{Q}} \rightarrow S_{\mathcal{R}}$ such that $\rho_{2} \circ \sigma_{\mathcal{Q}}=\sigma_{\mathcal{R}} \circ \rho_{1}$ and $\rho_{1}$ and $\rho_{2}$ are cellularly isotopic. Then if we replace the subdivision map $\sigma_{\mathcal{R}}$ by $\rho_{1} \circ \sigma_{\mathcal{Q}} \circ \rho_{1}^{-1}=\rho_{1} \circ \rho_{2}^{-1} \circ \sigma_{\mathcal{R}}$, then we get a new finite subdivision rule, which we still call $\mathcal{R}$, which has mesh approaching 0 . Furthermore, since $\rho_{1} \circ \rho_{2}^{-1}$ is cellularly isotopic to the identity map, by concatenating isotopies we still have an isotopy $H: \widehat{\mathbb{C}} \times I \rightarrow \widehat{\mathbb{C}}$ rel $P_{g}$ such that $H_{0}$ is the identity map, $H_{1}(\alpha)=\beta$, and $H_{1} \circ g$ is the subdivision map. Hence we can and do assume that $\mathcal{R}$ has mesh approaching 0 . This shows that $g$ is equivalent to the subdivision map $\sigma_{\mathcal{R}}$. To show that $g$ is a subdivision map of a finite subdivision rule, it suffices to show that $g$ and $\sigma_{\mathcal{R}}$ are conjugate, since then the finite subdivision rule $\mathcal{R}$ would pull back to a finite subdivision rule with subdivision map $g$.

We use the machinery of expansion complexes, developed in [4, to show that $\sigma_{\mathcal{R}}$ and $g$ are conjugate. By the choice of $n$, there is a point in $\widehat{\mathbb{C}}$ whose distance from $\beta$ is greater than $2 \epsilon$. This point is within $\epsilon$ of a vertex of $\mathcal{R}\left(S_{\mathcal{R}}\right)$, and the closed star in $\mathcal{R}\left(S_{\mathcal{R}}\right)$ of this vertex is in the interior of one of the two tiles of $S_{\mathcal{R}}$. By 4. Lemma 2.4], this tile of $S_{\mathcal{R}}$ is the seed of an expansion complex $X$ for $\mathcal{R}$. Let $h: X \rightarrow S_{\mathcal{R}}$ be the structure map for $X$, and let $\varphi$ be the expansion map. Then $X$ is homeomorphic to $\mathbb{R}^{2}$ and $\sigma_{\mathcal{R}} \circ h=h \circ \varphi$.

Since the restriction of $h$ to $h^{-1}\left(S_{\mathcal{R}} \backslash P_{g}\right)$ is a covering map, the maps $\left\{H_{t} \circ g: t \in I\right\}$ lift to a 1-parameter family $\left\{\Psi_{t}: t \in I\right\}$ of maps from $X$ to $X$ with $\Psi_{1}=\varphi$. Let $\Psi=\Psi_{0}$. Then $\Psi$ is a homeomorphism and $g \circ h=h \circ \Psi$. The standard conformal structure on $\widehat{\mathbb{C}}$ gives a nonsingular partial conformal structure on $S_{\mathcal{R}}$, and so this lifts to a conformal structure on $X$. Furthermore, $\Psi$ is conformal with respect to this lifted conformal structure. It now follows from [4, Theorems 6.6 and 6.7] that there is an $\mathcal{R}$-complex $X^{\prime}$ with structure map $h^{\prime}: X^{\prime} \rightarrow S_{\mathcal{R}}$ such that $X$ and $X^{\prime}$ have the same underlying space, $h^{\prime} \circ \rho=h$ where $\rho(x)=\lim _{n \rightarrow \infty} \Psi^{-n}\left(\varphi^{n}(x)\right)$, and $\Psi$ is the expansion map for $X^{\prime}$.

We prepare to show that if $x, y \in X$, then $h(x)=h(y)$ if and only if $h(\rho(x))=$ $h(\rho(y))$. For each positive integer $n$ set $\rho_{n}=\Psi^{-n} \circ \varphi^{n}$. By continuity it suffices to prove for every positive integer $n$ that if $x, y \in X$, then $h(x)=h(y)$ if and only if $h\left(\rho_{n}(x)\right)=h\left(\rho_{n}(y)\right)$. To begin the proof of this, let $k$ be a nonnegative integer, and let

and

$$
S_{\mathcal{R}}^{(k)}=\sigma_{\mathcal{R}}^{-k}\left(S_{\mathcal{R}} \backslash P_{g}\right)
$$

$$
X^{(k)}=h^{-1}\left(S_{\mathcal{R}}^{(k)}\right)
$$

The restriction of $h$ to $X^{(0)}$ is a covering projection onto $S_{\mathcal{R}}^{(0)}$, and the restriction of $H$ to $S_{\mathcal{R}}^{(0)} \times I$ lifts to a homotopy $X^{(0)} \times I \rightarrow X^{(0)}$ such that this homotopy 
extends to a homotopy $\widetilde{H}: X \times I \rightarrow X$ with $\widetilde{H}_{0}=$ id. We now have the following commutative diagram.

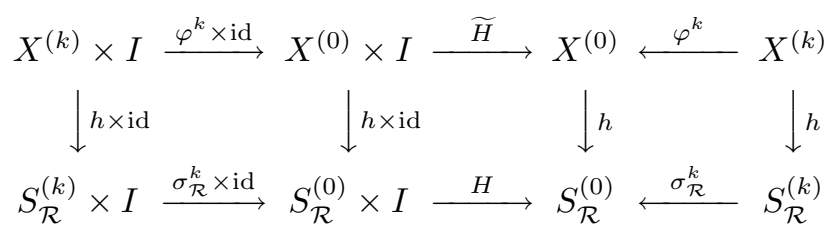

In this paragraph we show that $\varphi^{-k} \circ \widetilde{H}_{1} \circ \varphi^{k}$ is a lift of a map from $S_{\mathcal{R}}$ to $S_{\mathcal{R}}$. The restriction of $\sigma_{\mathcal{R}}^{k}$ to $S_{\mathcal{R}}^{(k)}$ is a covering projection onto $S_{\mathcal{R}}^{(0)}$ and the map $H \circ\left(\sigma_{\mathcal{R}}^{k} \times\right.$ id $)$ lifts to a homotopy $S_{\mathcal{R}}^{(k)} \times I \rightarrow S_{\mathcal{R}}^{(k)}$ which is the restriction of a homotopy $L: S_{\mathcal{R}} \times I \rightarrow S_{\mathcal{R}}$ with $L_{0}=$ id.

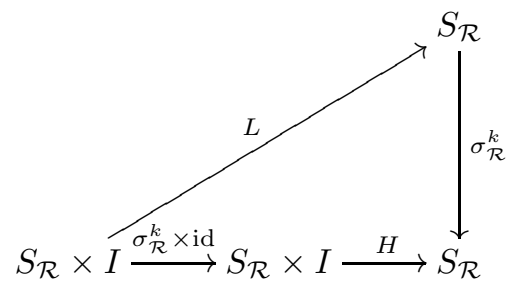

So both $L \circ(h \times$ id $)$ and $h \circ \varphi^{-k} \circ \widetilde{H} \circ\left(\varphi^{k} \times\right.$ id $)$ define continuous maps from $X^{(k)} \times I$ to $S_{\mathcal{R}}^{(k)}$ whose restrictions to $X^{(k)} \times\{0\}$ equal $h$ and

$$
\begin{aligned}
\sigma_{\mathcal{R}}^{k} \circ L \circ(h \times \mathrm{id}) & =H \circ\left(\sigma_{\mathcal{R}}^{k} \times \mathrm{id}\right) \circ(h \times \mathrm{id}) \\
& =h \circ \widetilde{H} \circ\left(\varphi^{k} \times \mathrm{id}\right) \\
& =h \circ \varphi^{k} \circ \varphi^{-k} \circ \widetilde{H} \circ\left(\varphi^{k} \times \mathrm{id}\right) \\
& =\sigma_{\mathcal{R}}^{k} \circ h \circ \varphi^{-k} \circ \widetilde{H} \circ\left(\varphi^{k} \times \mathrm{id}\right) .
\end{aligned}
$$

Hence $L \circ(h \times$ id $)=h \circ \varphi^{-k} \circ \widetilde{H} \circ\left(\varphi^{k} \times\right.$ id $)$, and so $\varphi^{-k} \circ \widetilde{H}_{1} \circ \varphi^{k}$ is a lift of $L_{1}$. Thus $\varphi^{-k} \circ \widetilde{H}_{1} \circ \varphi^{k}$ is a lift of a map from $S_{\mathcal{R}}$ to $S_{\mathcal{R}}$.

Next note that since $H_{1}: S_{\mathcal{R}} \rightarrow S_{\mathcal{R}}$ is a homeomorphism which is homotopic to the identity map rel $P_{g}$, so is its inverse. Let $G: S_{\mathcal{R}} \rightarrow S_{\mathcal{R}}$ be a homotopy rel $P_{g}$ from the identity map to $H_{1}^{-1}$. The argument of the previous paragraph with $G$ instead of $H$ shows that $\varphi^{-k} \circ \widetilde{G}_{1} \circ \varphi^{k}$ is a lift of a map from $S_{\mathcal{R}}$ to $S_{\mathcal{R}}$. Furthermore $\widetilde{H}_{1}=\left(\widetilde{G}_{1}\right)^{-1}$ and since $g=H_{1}^{-1} \circ \sigma_{\mathcal{R}}=G_{1} \circ \sigma_{\mathcal{R}}$, the case $k=1$ shows that $\Psi=\widetilde{G}_{1} \circ \varphi$ lifts $g$.

So

$$
\begin{aligned}
\rho_{n} & =\Psi^{-n} \circ \varphi^{n}=\left(\widetilde{G}_{1} \circ \varphi\right)^{-n} \circ \varphi^{n}=\left(\varphi^{-1} \circ\left(\widetilde{G}_{1}\right)^{-1}\right)^{n} \circ \varphi^{n} \\
& =\left(\varphi^{-1} \circ \widetilde{H}_{1} \circ \varphi\right) \circ\left(\varphi^{-2} \circ \widetilde{H}_{1} \circ \varphi^{2}\right) \circ \cdots \circ\left(\varphi^{-n} \circ \widetilde{H}_{1} \circ \varphi^{n}\right) .
\end{aligned}
$$

We have seen that every parenthesized function $F$ has the property that if $x, y \in X$ with $h(x)=h(y)$, then $h(F(x))=h(F(y))$. Hence $\rho_{n}$ also has this property. Similarly,

$$
\begin{aligned}
\rho_{n}^{-1} & =\varphi^{-n} \circ \Psi^{n}=\varphi^{-n} \circ\left(\widetilde{G}_{1} \circ \varphi\right)^{n} \\
& =\left(\varphi^{-n} \circ \widetilde{G}_{1} \circ \varphi^{n}\right) \circ \cdots \circ\left(\varphi^{-2} \circ \widetilde{G}_{1} \circ \varphi^{2}\right) \circ\left(\varphi^{-1} \circ \widetilde{G}_{1} \circ \varphi\right) .
\end{aligned}
$$


As for $\rho_{n}$, it follows that if $x, y \in X$ with $h(x)=h(y)$, then $h\left(\rho_{n}^{-1}(x)\right)=h\left(\rho_{n}^{-1}(y)\right)$. Thus if $x, y \in X$, then $h(x)=h(y)$ if and only if $h(\rho(x))=h(\rho(y))$.

The branched covering $h^{\prime}: X \rightarrow S_{\mathcal{R}}$ is a quotient map, and by the paragraph above, the map $h=h^{\prime} \circ \rho: X \rightarrow S_{\mathcal{R}}$ descends to the quotient space to a map $\mu: S_{\mathcal{R}} \rightarrow S_{\mathcal{R}}$. Similarly, the branched covering $h: X \rightarrow S_{\mathcal{R}}$ is a quotient map, and the map $h^{\prime}: X \rightarrow S_{\mathcal{R}}$ descends to the quotient space to a map $\mu^{\prime}: S_{\mathcal{R}} \rightarrow S_{\mathcal{R}}$. It is easily seen that $\mu^{\prime}=\mu^{-1}$. Note that

$$
g \circ \mu \circ h^{\prime}=g \circ h=h \circ \Psi=\mu \circ h^{\prime} \circ \Psi=\mu \circ \sigma_{\mathcal{R}} \circ h^{\prime} .
$$

Let $t_{1}$ and $t_{2}$ be the two open tiles of $S_{\mathcal{R}}$, and let $\widetilde{t_{1}}$ and $\widetilde{t_{2}}$ be components of their preimages under $h^{\prime}$. Since the restrictions of $h^{\prime}$ to $\widetilde{t_{1}}$ and to $\widetilde{t_{2}}$ are homeomorphisms, $g \circ \mu=\mu \circ \sigma_{\mathcal{R}}$ on $t_{1} \cup t_{2}$. Since $t_{1} \cup t_{2}$ is dense in $S_{\mathcal{R}}$, by continuity $g \circ \mu=\mu \circ \sigma_{\mathcal{R}}$ and so $g$ and $\sigma_{\mathcal{R}}$ are conjugate.

\section{Questions}

We close with a few questions.

Question 4.1. Is Theorem 1 true for all positive integers $n$, instead of just for $n$ sufficiently large? While the statements here and in [1] are for $n$ sufficiently large, we know of no obstruction for small values of $n$. It would be very interesting if the theorem were not true for all positive integers $n$.

Question 4.2. Is Theorem 1 true without the assumption that there are no periodic critical points? Most of the machinery for studying finite subdivision rules, including the expansion complex machinery used here, was developed for applications where the assumption of bounded valence was not restrictive. We do not know if the proof given here generalizes to the case where $f$ has periodic critical points.

\section{ACKNOWLEDGEMENT}

Our work, which is inspired by Sullivan's dictionary between rational maps and Kleinian groups, is part of an effort to further tie together Kleinian groups and rational maps. We thank Kevin Pilgrim for suggesting this problem to us and for numerous conversations about rational maps and finite subdivision rules.

\section{REFERENCES}

[1] M. Bonk and D. Meyer, Topological rational maps and subdivisions, in preparation.

[2] J. W. Cannon, W. J. Floyd, and W. R. Parry, Finite subdivision rules, Conform. Geom. Dyn. 5 (2001), 153-196 (electronic). MR.1875951 (2002j:52021)

[3] J. W. Cannon, W. J. Floyd, R. Kenyon, and W. R. Parry, Constructing rational maps from subdivision rules, Conform. Geom. Dyn. 7 (2003), 76-102 (electronic). MR1992038 (2004f:37062)

[4] J. W. Cannon, W. J. Floyd, and W. R. Parry, Expansion complexes for finite subdivision rules I, Conform. Geom. Dyn. 10 (2006), 63-99 (electronic). MR.2218641 (2007c:30048)

[5] J. W. Cannon and E. L. Swenson, Recognizing constant curvature groups in dimension 3, Trans. Amer. Math. Soc. 350 (1998), 809-849. MR1458317 (98i:57023)

[6] A. Douady and J. H. Hubbard, A proof of Thurston's topological characterization of rational functions, Acta Math. 171 (1993), 263-297. MR.1251582(94j:58143)

[7] J. Milnor, Dynamics in One Complex Variable: Introductory Lectures, Vieweg \& Sohn, Braunschweig/Wiesbaden, 1999. MR/1721240 (2002i:37057)

[8] K. M. Pilgrim, Canonical Thurston obstructions, Adv. Math. 158 (2001), 154-168. MR.1822682 (2001m:57004) 
[9] K. M. Pilgrim, Combinations of Complex Dynamical Systems, Springer Lecture Notes in Mathematics 1827, Springer-Verlag, Berlin Heidelberg New York, 2003. MR2020454 (2004m:37087)

[10] D. Sullivan, Quasiconformal homeomorphisms and dynamics I. Solution of the Fatou-Julia problem on wandering domains, Ann. Math. 122 (1985), 401-418. MR819553 (87i:58103)

[11] W. P. Thurston, Lecture notes, CBMS Conference, University of Minnesota at Duluth, 1983.

Department of Mathematics, Brigham Young University, Provo, Utah 84602

E-mail address: cannon@math.byu.edu

Department of Mathematics, Virginia Tech, Blacksburg, Virginia 24061

E-mail address: floyd@math.vt.edu

Department of Mathematics, Eastern Michigan University, Ypsilanti, Michigan 48197

E-mail address: walter.parry@emich.edu 